OUTP-99-68P

UG-FT-110/99

hep-ph/9912552

December 1999

\title{
A Three three-brane Universe: New Phenomenology for the New Millennium?
}

\author{
Ian I. Kogan ${ }^{a}$, Stavros Mouslopoulos ${ }^{a}$ ], Antonios Papazoglou ${ }^{a}$ ]

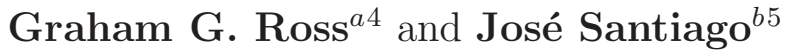 \\ ${ }^{a}$ Theoretical Physics, Department of Physics, Oxford University \\ 1 Keble Road, Oxford, OX1 3NP, UK \\ ${ }^{b}$ Department of Theoretical Physics, Granada University \\ Avenue Fuente Nueva, Granada, 18071, Spain
}

\begin{abstract}
We consider an extension of the Randall-Sundrum model with three parallel 3-branes in a 5 -dimensional spacetime. This new construction, apart from providing a solution to the Planck hierarchy problem, has the advantage that the SM fields are confined on a positive tension brane. The study of the phenomenology of this model reveals an anomalous first KK state which is generally much lighter than the remaining tower and also much more strongly coupled to matter. Bounds on the parameter space of the model can be placed by comparison of specific processes with the SM background as well as by the latest Cavendish experiments. The model suggests a further exotic possibility if one drops the requirement of solving the hierarchy problem. In this case gravity may result from the exchange of the ordinary graviton plus an ultralight KK state and modifications of gravity may occur at both small and extremely large scales.
\end{abstract}

\footnotetext{
${ }^{1}$ i.kogan@physics.ox.ac.uk

${ }^{2}$ s.mouslopoulos@physics.ox.ac.uk

3 a.papazoglou@physics.ox.ac.uk

${ }^{4}$ g.ross@physics.ox.ac.uk

5 jsantiag@ugr.es
} 


\section{Introduction}

Recently, there has been considerable interest in theories in which the SM fields are localized on a 3-brane in a higher dimensional spacetime. Depending on the dimensionality and the particular form of the geometry of this space, the long standing (Planck) hierarchy problem 9 can find alternative resolutions. Furthermore, these models make dramatical phenomenological predictions which can be directly confronted with current and future accelerator experiments as well as cosmological observations.

Antoniadis, Arkani-Hamed, Dimopoulos and Dvali [2, 3, 4] proposed that we live on a 3-brane in a $3+1+n$ space with fully factorizable geometry. The higher dimensional Planck scale $M$ is then related to the $4 \mathrm{D}$ Planck scale by $M_{\mathrm{Pl}}^{2}=M^{n+2} V_{n}$ where $V_{n}$ is the compactification volume. Taking the size of the new $n$ dimensions to be sufficiently large and identifying the $(4+n)$-Planck scale with the electroweak scale, an hierarchy between the electroweak and the Planck scale is introduced. Experimental and astrophysical constraints demand that $n \geq 2, M \gtrsim 30 \mathrm{TeV}$ and allow new dimensions even of submillimeter size. However, a new hierarchy must now be explained, namely the ratio of the large compactification radius to the electroweak scale. Even for six extra dimensions this must be greater than $10^{5}$.

In the light of this new problem, Randall and Sundrum proposed [5] an alternative scenario where they assumed one extra dimension along which the geometry is non-factorizable. Their construction consists of two parallel 3-branes sitting on the fixed points of an $S^{1} / Z_{2}$ orbifold (see Fig. 1). The 5D spacetime is essentially a slice of $A d S_{5}$ and the tensions of

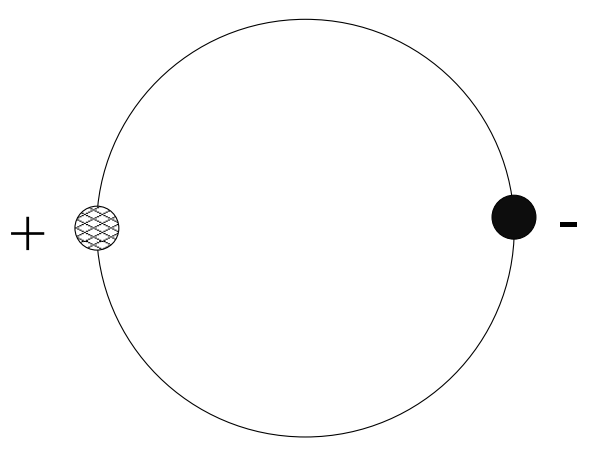

Figure 1: Randall-Sundrum Model with two branes at the orbifold $S^{1} / Z_{2}$ fixed points. Here and further a brane with positive cosmological constant is called positive or " $+{ }^{\prime \prime}$ brane and a brane with negative cosmological constant is called negative or "-" brane.

\footnotetext{
${ }^{6}$ i.e. the hierarchy between the Planck scale and the electroweak scale. There remains the problem of the hierarchy between the weak and gauge unification scales [1].
} 
the two 3-branes are chosen so that the 4D spacetime appears flat. This last requirement forces the one of the two branes to have negative tension. An exponential "warp" factor in the metric then generates a difference of the mass scales between the two branes that could be $\mathcal{O}\left(10^{15} \mathrm{GeV}\right)$ although the size of the orbifold is only of the order of Planck length. Assuming that the fundamental mass scale on the positive brane is of the order of $M_{\mathrm{Pl}}$ we can readily get a mass scale on the negative brane of the order the electroweak scale, thus solving the hierarchy problem. In this the compactification radius need only be some 35 times larger than the Planck length. The phenomenology of this model has been extensively explored in Refs. [6, 7]. The KK tower of spin-2 graviton resonances starts from the $\mathrm{TeV}$ scale with TeV spacing giving rise to characteristic signals in high energy colliders.

Several models have been constructed since then [8, 9, 10] that extend the original RS model to multibrane configurations, parallel or intersecting, with a single or different cosmological constants between them. A generic characteristic of these models is the presence of negative tension branes which are necessary for the branes to be flat. Interesting cases where the branes are not flat or the extra dimension(s) is not compact have also been considered in Refs. [11, 12, 13, 14, 15]. The phenomenology of these models is generally complicated and little has been written about them.

For a viable theory the "brane world" must reproduce correct gravity and cosmology of our Universe. In the RS picture the negative cosmological constant of the bulk is used to cancel the cosmological constant or tension on the brane. On a brane with a positive tension (as for example in the single brane scenario [13]), gravity is effectively confined to the brane by the steep "warp" factor generated by the tension dominating the brane. Of course in realistic cosmologies the energy density of the Universe must be dominated by matter. An important observation about the cosmology of the "brane world" was made in Refs. [16, 17, 18]. These papers showed that the Hubble parameter $H$ governing the expansion of the scale factor on the brane has a different behavior than derived from the usual 4-d Friedmann equations. In particular, the Hubble parameter is proportional to the energy density on the brane instead of the familiar dependence $H \sim \sqrt{\rho}$.

In the papers [19, 20, 21, 22] (see also [23]) it was shown that for the Randall-Sundrum construction the full energy density $\rho$ is a sum of a vacuum energy density, i.e. brane tension $\Lambda_{b r}$ and a matter energy density $\rho_{m}$ and the correct expression for Hubble constant squared can be obtained by cancelling the leading $\left(\Lambda_{b r}+\rho_{m}\right)^{2}$ term with the term $\Lambda_{B}^{2}$ coming from the negative bulk cosmological constant $\Lambda_{B}$ so that $H \sim \sqrt{\Lambda_{b r} \rho_{m}}$. From this picture one can immediately see that to live on a negative brane is impossible - we either have normal matter with positive energy density, but imaginary Hubble constant, or real Hubble constant, but negative energy density, i.e. antigravity. Because we are living in the Universe with a real Hubble constant and without any noticeble effects of antigravity the negative brane as a model of our world must be excluded. It can be shown that one may 
have normal cosmology on a negative brane in more general models with nontrivial bulk stress-energy tensor [24, 25] but it puts extra constraints on parameters of the model and in the typical case life on a negative brane leads to the same dilemma - either antigravity or imaginary Hubble constant.

The purpose of this paper is to formulate a new model of a "brane world" in which we live on a positive brane thus avoiding these cosmological problems. The model consists of two positive branes located at the fixed points of a $S_{1} / Z_{2}$ orbifold with one negative brane which can move freely in between (see Fig. 2). It is easy to see that the two-brane RS

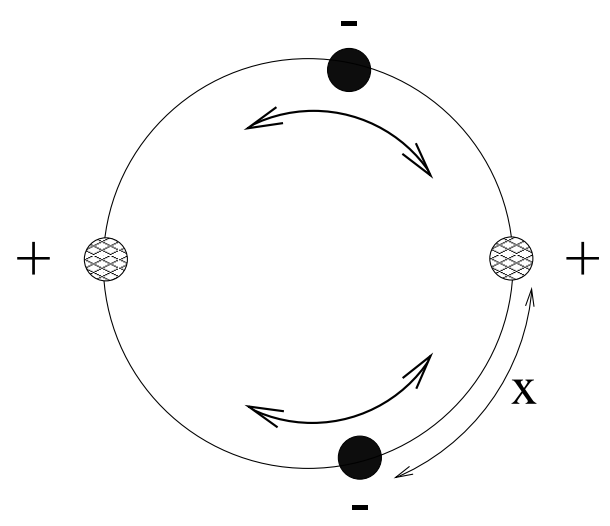

Figure 2: $\quad+-+$ model with two " + " branes at the fixed points and moving " - " branes. In the limiting case when $x \rightarrow 0$ we have a RS configuration.

model is nothing but the limiting case of our three-brane model, when the negative brane hits one of the possitive branes. The model has three parameters, the bulk curvature, the warp factor and the $x$ factor - which effectively measures the distance between one of the positive branes and the negative brane. The RS model corresponds to the limiting case $x=0$.

Let us note that so far it is unclear what are the selection rules on branes at orbifold fixed points and moving branes. In a heterotic M-theory for example [26] one can think about boundaries of eleven-dimensional space after compactification on a suitable CalabiYau manifold as negative branes where a fundamental five-brane in M-theory after wrapping on 2-cycles may play the role of a " + " brane. Moving a " + " brane towards one of the ${ }^{\prime \prime}-{ }^{\prime \prime}$ branes we can transform it into a " + " brane, so one can get a configuration of the type ++ - or with more than one moving " + " brane - the only constraint is the that the sum of all "charges" is zero. Let us note that each moving brane on an orbifold must be counted with a double charge because of it mirror image. Ø It is unclear if one may have

\footnotetext{
${ }^{7}$ We are grateful to Andre Lucas for interesting discussion on this subject.
} 


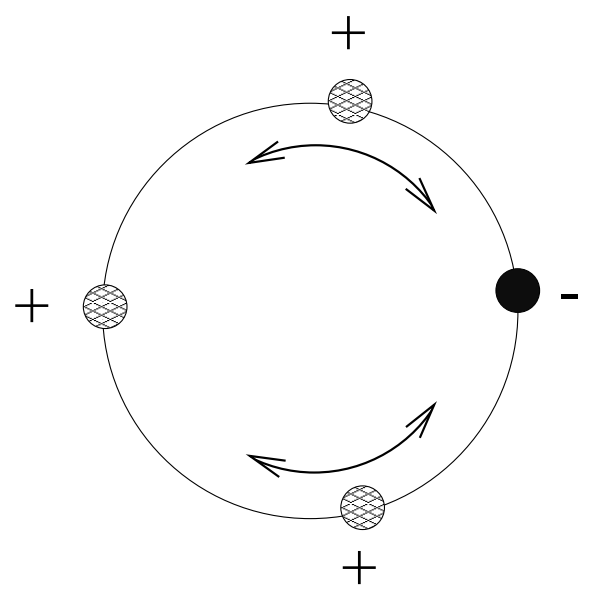

Figure 3: ++- model which is a RS configuration on Figure 1 with an insertion of a moving " + " brane. This configuration can be obtained from a configuration with only " $-"$ branes at the orbifold points and several moving " + " branes.

moving "-" branes. At the same time it is necessary to say that so far the full string/Mtheory description of these multibrane configurations is missing and we can not exclude a possibility that there are moving negative branes.

In this paper we shall discuss in details the +-+ model of Fig.2. One of the striking predictions of our model is the fact that the first KK mode can be very light and strongly coupled compared to the rest of the KK states, so that the phenomenology is determined mostly by it. An unusual possibility arises if we relax the requirement that the Planck hierarchy problem is solved. In this case this light mode can be so light that the corresponding wavelength can be by order of $1 \%$ of the observable size of the Universe while the second KK mode is in submillimeter region. Surprisingly enough this situation is not excluded experimentally! Thus one may have "Bi-Gravity" - in all experimentally analyzed regions gravitational attraction is due to an exchange of two particles - the massless graviton and ultralight first KK mode. Only at scales larger than $10^{26} \mathrm{~cm}$ will the first $\mathrm{KK}$ mode decouple leading to a much smaller gravitational coupling beyond this length scale.

The reason the anomalously light KK mode exists is due to the fact that with more that one " + " brane there will be a bound state on each of them when they have infinite separation. At finite distances there is a mixing between the two localized states. One superposition is the true ground state while the other configuration has non-zero mass, but the gap may be very small - it is given by a tunneling factor. This effect takes place not only in +-+ model but in ++- and other models with more than two " + " branes (in which case there may be more than one light mode). In this paper we consider only the 
+-+ configuration which has the interesting "Bi-Gravity" possibility. Other models will be considered in separate publications.

The organisation of the paper is as follows. In the next Section we construct the model and discuss the spectrum of KK excitations. In Section 3 the first and subsequent KK modes are considered in more detail and their masses and couplings as a function of the "warp" factor and the parameter $x$ are discussed using both analytical and numerical methods. In Section 4 we discuss the phenomenology when the first KK mode has a mass in the meV$\mathrm{TeV}$ region. In Section 5 we discuss the unusual "Bi-Gravity" scenario when the Compton wavelength of the first KK mode is by order of $10^{26} \mathrm{~cm}$ while the Compton wavelength of a second KK mode is less than $1 \mathrm{~mm}$. In conclusion, we discuss the possible generalization of our model and questions for future investigation.

\section{The 3-brane model}

Our model consists of three parallel 3-branes in an $A d S_{5}$ space with cosmological constant $\Lambda<0$. The 5 -th dimension has the geometry of an orbifold and the branes are located at $L_{0}=0, L_{1}$ and $L_{2}$ where $L_{0}$ and $L_{2}$ are the orbifold fixed points (see Fig.2). Firstly we consider the branes having no matter on them in order to find a suitable vacuum solution. The action of this setup is:

$$
S=\int d^{4} x \int_{-L_{2}}^{L_{2}} d y \sqrt{-G}\left\{-\Lambda+2 M^{3} R\right\}-\sum_{i} \int_{y=L_{i}} d^{4} x V_{i} \sqrt{-\hat{G}^{(i)}}
$$

where $\hat{G}_{\mu \nu}^{(i)}$ is the induced metric on the branes and $V_{i}$ their tensions. The notation is the same as in Ref. [5]. The Einstein equations that arise from this action are:

$$
R_{M N}-\frac{1}{2} G_{M N} R=-\frac{1}{4 M^{3}}\left(\Lambda G_{M N}+\sum_{i} V_{i} \frac{\sqrt{-\hat{G}^{(i)}}}{\sqrt{-G}} \hat{G}_{\mu \nu}^{(i)} \delta_{M}^{\mu} \delta_{N}^{\nu} \delta\left(y-L_{i}\right)\right)
$$

At this point we demand that our metric respects 4D Poincaré invariance. The metric ansatz with this property is the following:

$$
d s^{2}=e^{-2 \sigma(y)} \eta_{\mu \nu} d x^{\mu} d x^{\nu}+d y^{2}
$$

Here the "warp" function $\sigma(y)$ is essentially a conformal factor that rescales the 4D component of the metric. A straightforward calculation gives us the following differential equations for $\sigma(y)$ :

$$
\begin{aligned}
\left(\sigma^{\prime}\right)^{2} & =k^{2} \\
\sigma^{\prime \prime} & =\sum_{i} \frac{V_{i}}{12 M^{3}} \delta\left(y-L_{i}\right)
\end{aligned}
$$


where $k=\sqrt{\frac{-\Lambda}{24 M^{3}}}$ is a measure of the curvature of the bulk.

The solution of these equations consistent with the orbifold geometry is precisely:

$$
\sigma(y)=k\left\{L_{1}-|| y\left|-L_{1}\right|\right\}
$$

with the requirement that the brane tensions are tuned to $V_{0}=-\Lambda / k>0, V_{1}=\Lambda / k<0$, $V_{2}=-\Lambda / k>0$. If we consider massless fluctuations of this vacuum metric as in Ref. [5] and then integrate over the 5-th dimension, we find the 4D Planck mass is given by

$$
M_{\mathrm{Pl}}^{2}=\frac{M^{3}}{k}\left[1-2 e^{-2 k L_{1}}+e^{-2 k\left(2 L_{1}-L 2\right)}\right]
$$

The above formula tells us that for large enough $k L_{1}$ and $k\left(2 L_{1}-L_{2}\right)$ the three mass scales $M_{\mathrm{Pl}}, M, k$ can be taken to be of the same order. Thus we take $k \sim \mathcal{O}(M)$ in order not to introduce a new hierarchy, with the additional restriction $k<M$ so that the bulk curvature is small compared to the 5D Planck scale so that we can trust our solution. Furthermore, if we put matter on the third brane all the physical masses $m$ on the third brane will be related to the mass parameters $m_{0}$ of the fundamental $5 \mathrm{D}$ theory by the conformal (warp) factor

$$
m=e^{-\sigma\left(L_{2}\right)} m_{0}=e^{-k\left(2 L_{1}-L_{2}\right)} m_{0}
$$

Thus we can assume that the third brane is our universe and get a solution of the Planck hierarchy problem arranging $e^{-k\left(2 L_{1}-L_{2}\right)}$ to be of $\mathcal{O}\left(10^{-15}\right)$, i.e $2 L_{1}-L_{2} \approx 35 k^{-1}$. In this case all the parameters of the model $L_{1}^{-1}, L_{2}^{-1}$ and $\mathrm{k}$ are of the order of Plank scale.

To determine the phenomenology of the model we need to know the KK spectrum that follows from the dimensional reduction. This is determined by considering the (linear) fluctuations of the metric of the form:

$$
d s^{2}=\left[e^{-2 \sigma(y)} \eta_{\mu \nu}+\frac{2}{M^{3 / 2}} h_{\mu \nu}(x, y)\right] d x^{\mu} d x^{\nu}+d y^{2}
$$

Here we have ignored the dilaton mode that could be used to stabilize the brane positions $L_{1}$ and $L_{2}$ as discussed in Refs. [25, 27, 28, 29]. While the dilaton and its KK excitations may lead to additional observable phenomena its inclusion should not change the phenomenology of the graviton KK modes. We have also ignored the off diagonal vector KK modes simply because they don't couple to the SM fields.

We expand the field $h_{\mu \nu}(x, y)$ in graviton and KK states plane waves:

$$
h_{\mu \nu}(x, y)=\sum_{n=0}^{\infty} h_{\mu \nu}^{(n)}(x) \Psi^{(n)}(y)
$$

where $\left(\partial_{\kappa} \partial^{\kappa}-m_{n}^{2}\right) h_{\mu \nu}^{(n)}=0$ and fix the gauge as $\partial^{\alpha} h_{\alpha \beta}^{(n)}=h_{\alpha}^{(n) \alpha}=0$. The function $\Psi^{(n)}(y)$ will obey a second order differential equation which after a change of variables reduces to 
an ordinary Schrödinger equation:

$$
\begin{gathered}
\left\{-\frac{1}{2} \partial_{z}^{2}+V(z)\right\} \hat{\Psi}^{(n)}(z)=\frac{m_{n}^{2}}{2} \hat{\Psi}^{(n)}(z) \\
\text { with } \quad V(z)=\frac{15 k^{2}}{8[g(z)]^{2}}-\frac{3 k}{2 g(z)}\left[\delta(z)+\delta\left(z-z_{2}\right)-\delta\left(z-z_{1}\right)-\delta\left(z+z_{1}\right)\right]
\end{gathered}
$$

The new variables and wavefunction in the above equation are defined as:

$$
z \equiv\left\{\begin{array}{cl}
\frac{2 e^{k L_{1}-e^{2 k L_{1}-k y}-1}}{k} & y \in\left[L_{1}, L_{2}\right] \\
\frac{e^{k y}-1}{k} & y \in\left[0, L_{1}\right] \\
-\frac{e^{-k y}-1}{k} & y \in\left[-L_{1}, 0\right] \\
-\frac{2 e^{k L_{1}-e^{2 k L_{1}+k y}-1}}{k} & y \in\left[-L_{2},-L_{1}\right] \\
\hat{\Psi}^{(n)}(z) \equiv \Psi^{(n)}(y) e^{\sigma / 2}
\end{array}\right.
$$

and the function $g(z)$ as $g(z) \equiv k\left\{z_{1}-|| z\left|-z_{1}\right|\right\}+1$, where $z_{1}=z\left(L_{1}\right)$.

This is a quantum mechanical problem with $\delta$-function potentials of different weight and an extra $1 / g^{2}$ smoothing term (due to the AdS geometry) that gives the potential a double "volcano" form. The change of variables has been chosen so that there are no first derivative terms in the differential equation.

An interesting characteristic of this potential is that it always gives rise to a (massless) zero mode which reflects the fact that Lorentz invariance is preserved in $4 \mathrm{D}$ spacetime. It is given by

$$
\hat{\Psi}^{(0)}=\frac{A}{[g(z)]^{3 / 2}}
$$

The normalization factor $A$ is determined by the requirement $\int_{0}^{z_{2}} d z\left[\hat{\Psi}^{(0)}(z)\right]^{2}=1$, chosen so that we get the standard form of the Fierz-Pauli Lagrangian.

In the specific case where $L_{1}=L_{2} / 2$ (and with zero hierarchy) the potential and thus the zero mode's wavefunction is symmetric with respect to the second brane. When the second brane moves towards the third one the wavefunction has a minimum on the second brane but different heights on the other two branes, the difference generating the hierarchy between the first and the third brane.

For the KK modes the solution is given in terms of Bessel functions. For $y$ lying in the regions $\mathbf{A} \equiv\left[0, L_{1}\right]$ and $\mathbf{B} \equiv\left[L_{1}, L_{2}\right]$, we have:

$$
\hat{\Psi}^{(n)}\left\{\begin{array}{l}
\mathbf{A} \\
\mathbf{B}
\end{array}\right\}=\sqrt{\frac{g(z)}{k}}\left[\left\{\begin{array}{l}
A_{1} \\
B_{1}
\end{array}\right\} J_{2}\left(\frac{m_{n}}{k} g(z)\right)+\left\{\begin{array}{c}
A_{2} \\
B_{2}
\end{array}\right\} Y_{2}\left(\frac{m_{n}}{k} g(z)\right)\right]
$$


The boundary conditions (one for the continuity of the wavefunction at $z_{1}$ and three for the discontinuity of its first derivative at $0, z_{1}, z_{2}$ ) result in a $4 \times 4$ homogeneous linear system which, in order to have a non-trivial solution, leads to the vanishing determinant:

$$
\left|\begin{array}{cccc}
J_{1}\left(\frac{m}{k}\right) & Y_{1}\left(\frac{m}{k}\right) & 0 & 0 \\
0 & 0 & J_{1}\left(\frac{m}{k} g\left(z_{2}\right)\right) & Y_{1}\left(\frac{m}{k} g\left(z_{2}\right)\right) \\
J_{1}\left(\frac{m}{k} g\left(z_{1}\right)\right) & Y_{1}\left(\frac{m}{k} g\left(z_{1}\right)\right) & J_{1}\left(\frac{m}{k} g\left(z_{1}\right)\right) & Y_{1}\left(\frac{m}{k} g\left(z_{1}\right)\right) \\
J_{2}\left(\frac{m}{k} g\left(z_{1}\right)\right) & Y_{2}\left(\frac{m}{k} g\left(z_{1}\right)\right) & -J_{2}\left(\frac{m}{k} g\left(z_{1}\right)\right) & -Y_{2}\left(\frac{m}{k} g\left(z_{1}\right)\right)
\end{array}\right|=0
$$

(where we have suppressed the subscript $n$ on the masses $m_{n}$ )

This is essentially the mass quantization condition which gives the spectrum of the KK states. For each mass we can then determine the wave function with normalization $\int_{0}^{z_{2}} d z\left[\hat{\Psi}^{(n)}(z)\right]^{2}=1$

From the form of the potential we can immediately deduce that there is a second "bound" state, the first KK state. In the symmetric case, $L_{1}=L_{2} / 2$, this is simply given by reversing the sign of the graviton wave function for $y>L_{1}$ (it has one zero at $\left.L_{1}\right)$. When the second brane moves towards the third this symmetry is lost and the first KK wave function has a very small value on the first brane, a large value on the third and a zero very close to the first brane.

The interaction of the KK states to the SM particles is found as in Ref. [30] by expanding the minimal gravitational coupling of the SM Lagrangian $\int d^{4} x \sqrt{-\hat{G}} \mathcal{L}(\hat{G}, S M$ fields $)$ with respect to the metric. After the rescaling due to the "warp" factor we get:

$$
\begin{aligned}
\mathcal{L}_{i n t} & =-\frac{g\left(z_{2}\right)^{3 / 2}}{M^{3 / 2}} \sum_{n \geq 0} \hat{\Psi}^{(n)}\left(z_{2}\right) h_{\mu \nu}^{(n)}(x) T_{\mu \nu}(x)= \\
& =-\frac{A}{M^{3 / 2}} h_{\mu \nu}^{(0)}(x) T_{\mu \nu}(x)-\sum_{n>0} \frac{\hat{\Psi}^{(n)}\left(z_{2}\right) g\left(z_{2}\right)^{3 / 2}}{M^{3 / 2}} h_{\mu \nu}^{(n)}(x) T_{\mu \nu}(x)
\end{aligned}
$$

with $T_{\mu \nu}$ the energy momentum tensor of the SM Lagrangian. Thus the coupling suppression of the zero and KK modes to matter is respectively:

$$
\begin{aligned}
\frac{1}{c_{0}} & =\frac{A}{M^{3 / 2}} \\
\frac{1}{c_{n}} & =\frac{\hat{\Psi}^{(n)}\left(z_{2}\right) g\left(z_{2}\right)^{3 / 2}}{M^{3 / 2}}
\end{aligned}
$$

For the zero mode the normalization constant $A$ is $\frac{M^{3 / 2}}{M_{\mathrm{P} 1}}$ which gives the Newtonian gravitational coupling suppression $c_{0}=M_{\mathrm{Pl}}$. 


\section{The first and subsequent KK modes: Masses and coupling constants}

As discussed above the KK spectrum has a special first mode which for all $x$ significantly different from unity has very different behaviour compared to the other KK states. In the case $x>>1$ we may obtain a reliable approximation to its mass by using the first terms of the Bessel power series. From now on we shall use a convenient choice of parameters: the measure of the curvature of the bulk $k$, the separation of the second and third brane $x=k\left(L_{2}-L_{1}\right)$ and the hierarchy factor $w=e^{-k\left(2 L_{1}-L_{2}\right)}$. The first KK mode has mass given by

$$
m_{1}=2 k w e^{-2 x}
$$

The normalization integral can also then be done analytically and the coupling suppression is found to be independent of $x$ and equal to

$$
c_{1}=w M_{\mathrm{Pl}}
$$

The reason for this is readily understood because, being dominantly a bound state of the volcano potential on our brane, it is largely localized on it.

The masses of the other KK states in the above region are found to depend in a different way on the parameter $x$. Numerically we find out that the mass of the second state and the spacing $\Delta m$ between the subsequent states have the form:

$$
\begin{aligned}
m_{2} & \approx 4 k w e^{-x} \\
\Delta m & \approx \varepsilon k w e^{-x}
\end{aligned}
$$

where $\varepsilon$ is a number between 1 and 2. The spacing only approaches a constant for high enough levels when the Bessel arguments become much greater than one. Again solving numerically, we find the couplings of the higher modes are suppressed relative to the lowest mode by a factor proportional to $e^{x}$. To illustrate the difference between the first and second modes at large $x$, we consider the case $w=e^{-35}, x=5$ and $k=10^{17} \mathrm{GeV}$. The masses and coupling suppressions of the first two modes are

$$
\begin{aligned}
& m_{1} \approx 5.7 \mathrm{MeV} \text { with } c_{1}=630 \mathrm{GeV} \\
& m_{2} \approx 1.6 \mathrm{GeV} \quad \text { with } \quad c_{2}=52500 \mathrm{GeV}
\end{aligned}
$$

When the second brane approaches the third brane, the above approximations break down and the first mode is not so different from the others. Its mass rises above $100 \mathrm{GeV}$ and the coupling is no longer constant but has a small dependence on $x$. In the extreme case where the positions of the second and the third brane coincide, the positive brane 


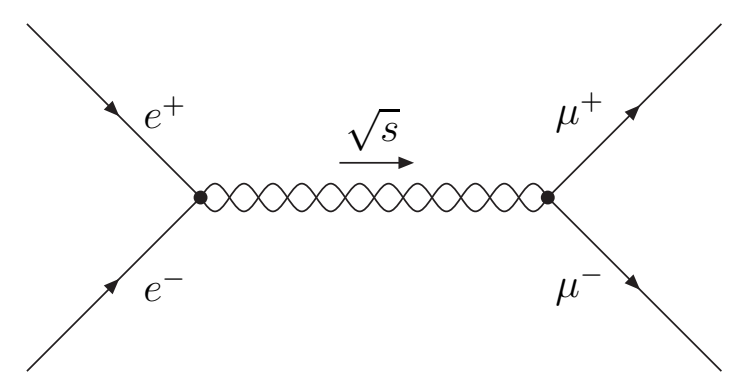

Figure 4: $e^{+} e^{-} \rightarrow \mu^{+} \mu^{-}$

disappears and we obtain the original Randall-Sundrum model. In this case the spectrum starts from several hundreds of $\mathrm{GeV}$ up to some $\mathrm{TeV}$ depending on the choice of $k$. It has large spacing between the KK states and TeV coupling suppression.

\section{Phenomenology}

In this Section we will present a brief discussion of the phenomenology of the KK modes to be expected in colliders, concentrating on the simple process $e^{+} e^{-} \rightarrow \mu^{+} \mu^{-}$. The analysis is readily generalized to include $q \bar{q}, g g$ initial and final states. A more complete discussion will appear elsewhere.

Using the Feynman rules of Ref. [30] the contribution of the KK modes to $e^{+} e^{-} \rightarrow \mu^{+} \mu^{-}$ is given by

$$
\sigma\left(e^{+} e^{-} \rightarrow \mu^{+} \mu^{-}\right)=\frac{s^{3}}{1280 \pi}|D(s)|^{2}
$$

where $D(s)$ is the sum over the propagators multiplied by the appropriate coupling suppressions:

$$
D(s)=\sum_{n>0} \frac{1 / c_{n}^{2}}{s-m_{n}^{2}+i \Gamma_{n} m_{n}}
$$

and $s$ is the C.M. energy of $e^{+} e^{-}$.

Note that the bad high energy behaviour of this cross section is expected since we are working with an effective - low energy non-renormalizable theory of gravity. Our effective theory is valid up to an energy scale $M_{s}$ (which is $\mathcal{O}(\mathrm{TeV})$ ), which acts as an ultraviolet cutoff. The theory that is valid above this scale is supposed to give a consistent description of quantum gravity. Since this is unknown we are only able to detemine the contributions of the KK states with masses less than this scale. This means that the summation in the previous formula should stop at the KK mode with mass near the cutoff. 
The decay rates of the KK states that are present in the above formula are given by:

$$
\Gamma_{n}=\beta \frac{m_{n}^{3}}{c_{n}^{2}}
$$

where $\beta$ is a dimensionless constant that is between $\frac{9}{80 \pi} \approx 0.035$ (in the case that the KK is light enough, i.e. smaller than $0.5 \mathrm{MeV}$, that can decay only to massless gauge bosons) and $\frac{37}{192 \pi} \approx 0.061$ (in the case where the KK is heavy enough that can decay to all SM particles).

The details of computation of the total cross section depends on the KK spectrum. In the case that $x$ is small, $x \lesssim 5$, we have a widely spaced discrete spectrum (from the point of view of $\mathrm{TeV}$ physics) close to the one of the Randall-Sundrum case with cross section at a KK resonances of the form $\sigma_{\text {res }} \sim s^{3} / m_{n}^{8}$. To be definite we will take $k=10^{17} \mathrm{GeV}$, $w=e^{-35}$. In the limit of very low $x(x \lesssim 0.1)$ the mass of the first mode is above current experimental energies $\left(m_{1} \sim 200 \mathrm{GeV}\right)$ and the phenomenology is that discussed in [6]. For the rest of the discrete spectrum region $(0.1 \lesssim x \lesssim 5)$ however there are always KK

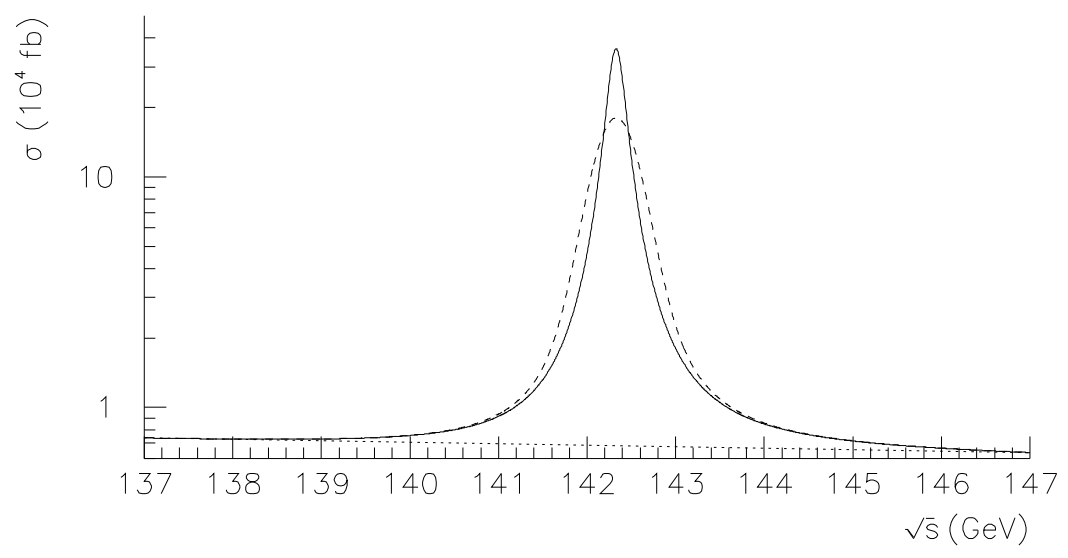

Figure 5: $e^{+} e^{-} \rightarrow \mu^{+} \mu^{-}$Resonance of the third mode for $k=10^{17} \mathrm{GeV}, w=e^{-35}$ and $x=1$. The higher curve is the theoretical curve, the smaller one is the result of the smoothing by the convolution of the theoretical curve and the experimental resolution of the beam. The SM background lies below them.

resonances in the range of energies of collider experiments. Due to the uncertainty in the collision energy the narrow KK peak will be reduced as shown in Fig. 5. In this case the contribution of the KK modes gives an excess of $\sim 10 \%$ over the SM contribution which 
would have been seen either by direct scanning if the resonance is near the energy at which the experiments actually run or by means of the process $e^{+} e^{-} \rightarrow \gamma \mu^{+} \mu^{-}$which scans a continuum of energies below the center of mass energy of the experiment. Thus this range of $k, w$, and $x$ is already excluded. Of course if $k$ is raised the KK modes become heavier and there will be a value for which the lightest KK mode is above the experimental limits. However as $k$ increases it reintroduces the hierarchy problem which the warp factor is designed to eliminate.

For values of $\mathrm{x}$ greater than $x \sim 5$ the spacing in the spectrum is so small that we can safely consider it to be continuous. In this case we substitute in $D(s)$ the sum for $n \geq 2$ by an integral over the mass of the KK excitations, i.e.

$$
D(s)_{K K} \approx \frac{1 / c_{1}^{2}}{s-m_{1}^{2}+i \Gamma_{1} m_{1}}+\frac{1}{\Delta m c^{2}} \int_{m_{2}}^{M_{s}} d m \frac{1}{s-m^{2}+i \epsilon}
$$

where the value of the integral is $\sim i \pi / 2 \sqrt{s}$ with the principal value negligible in the region of interest $\left(\sqrt{s} \ll M_{s}\right)$ and we have considered constant coupling suppression $c$ for the modes with $n \geq 2$. The first state is singled out because of its different coupling. In fact at these values of $x$ the biggest contribution comes from this state (the coupling of the rest being very small). For $w \approx e^{-35}$ the contribution of the KK tower is negligible

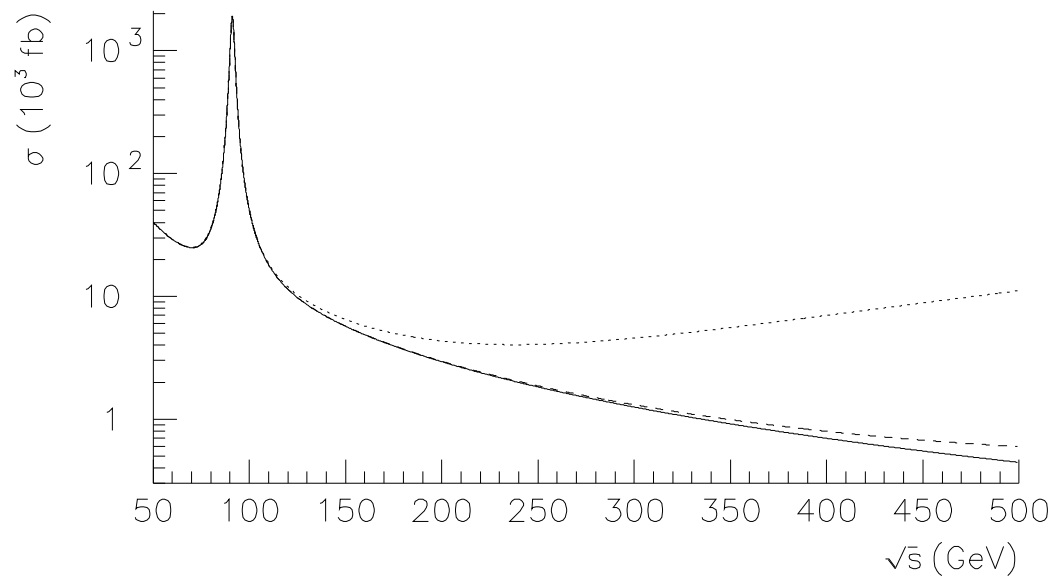

Figure 6: $e^{+} e^{-} \rightarrow \mu^{+} \mu^{-}$Cross section in the continuum limit for $k=10^{17} \mathrm{GeV}$ and $x=11$. The upper curve is for $w=e^{-36}$, the medium one for $w=e^{-35}$ and the lower one is the SM contribution (one loop calculation). The resonance we see is the $\mathrm{Z}$ peak.

compared with the SM background. However if we allow the value of $w$ to decrease, the 
coupling of the first state increases enough to give noticeable contribution to the cross section $\left(\sim 30 \%\right.$ at $\sqrt{s}=189 \mathrm{GeV}$ for $\left.w \approx e^{-36}\right)$. Thus we can exclude in this continuum limit values of $w \approx e^{-36}$ and below. A quantitative estimate is given in Fig. 6 where three curves are shown, the lowest one corresponds to the SM alone, the second takes into account also the tower of $\mathrm{KK}$ in the case of $w=e^{-35}$ and the last one is also with the KK tower but this time with $w=e^{-36}$ which as can be seen from the plot is already excluded.

To summarize, from the process $e^{+} e^{-} \rightarrow \mu^{+} \mu^{-}$we can exclude a window of the form $0.1 \lesssim x \lesssim 5$ for the preferred value of $w$ (this window corresponds to a value of the mass of the first KK mode between $\sim 0.3 \mathrm{GeV}$ and $\sim 200 \mathrm{GeV}$ ). Both the the very low $x$ limit and the continuum limit are allowed. Finally, we can also exclude values of the hierarchy greater than $\sim e^{-36}$ except for the $0 \leq x \lesssim 0.1$ region.

We have considered as an example the process $e^{+} e^{-} \rightarrow \mu^{+} \mu^{-}$but it may be possible to obtain similar or even stricter bounds could be obtained from other processes like $e^{+} e^{-} \rightarrow \gamma+$ missing energy for example. We shall present the detailed cross-section of the process $e^{+} e^{-} \rightarrow \gamma+$ light KK mode in another publication, let us only mention here that by dimensional analysis the ratio of this cross-section to two-photon electron-positron annihilation

$$
\frac{\sigma\left(e^{+} e^{-} \rightarrow \gamma+\text { light KK mode }\right)}{\sigma\left(e^{+} e^{-} \rightarrow \gamma+\gamma\right)} \sim \frac{s}{c_{1}^{2} \alpha} \sim 10^{2} \frac{s}{c_{1}^{2}}
$$

and so we see that this process may be phenomenologically very important.

A further bound on the parameters of our model can be put from the Cavendish experiments. The fact that gravity is Newtonian at least up to millimeter distances implies that the corrections to gravitational law due to the presence of the KK states must be negligible for such distances. The gravitational potential is the Newton law plus a Yukawa potential due to the exchange of the KK massive particles (in the Newtonian limit):

$$
V(r)=-\frac{1}{M_{\mathrm{Pl}}^{2}} \frac{M_{1} M_{2}}{r}\left(1+\sum_{n>0}\left(\frac{M_{\mathrm{Pl}}}{c_{n}}\right)^{2} e^{-m_{n} r}\right)
$$

The contribution to the above sum of the second and higher modes is negligible compared with the one of the first KK state, because they have larger masses and coupling suppressions. Thus, the condition for the corrections of the Newton law to be small for millimeter scale distances is:

$$
x<15-\frac{1}{2} \ln \left(\frac{-\ln w}{k w} \mathrm{GeV}\right)
$$

The logarithm for any reasonable choice of the parameters $k, w$ gives a contribution $\mathcal{O}(1)$, so we can safely say that $x \approx 15$ is the maximum brane separation allowed.

Combining the above results the allowed region of the parameters of our model for $k=10^{17} \mathrm{GeV}$ and $w=e^{-35}$ are shown in Fig. [1. 


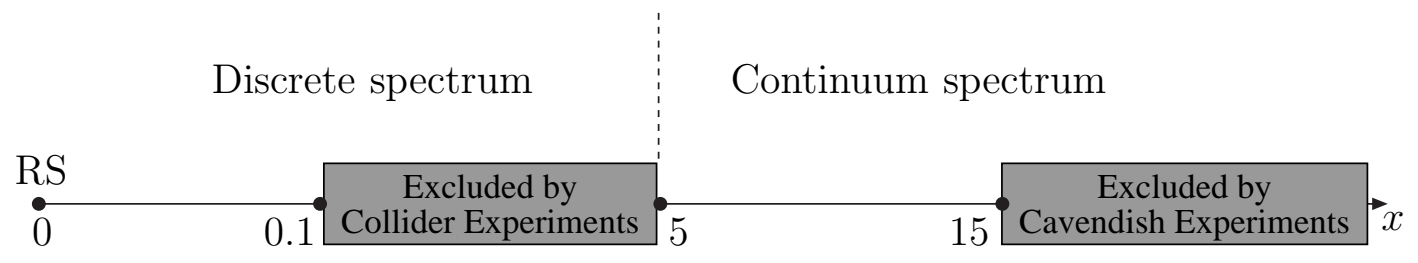

Figure 7: Excluded regions for $k=10^{17} \mathrm{GeV}$ and $w=e^{-35}$

\section{Bi-Gravity}

Equations (21) and (24) show that, for large $x$, the lightest KK mode splits off from the remaining tower. This leads to an exotic possibility in which the lightest KK mode is the dominant source of Newtonian gravity!

Cavendish experiments and astronomical observations studying the motions of distant galaxies have put Newtonian gravity to test from submillimeter distances up to distances that correspond to $1 \%$ of the size of observable Universe, searching for violations of the weak equivalence principle and inverse square law. In the context of the graviton KK modes discussed above this constrains $m<10^{-31} \mathrm{eV}$ or $m>10^{-4} \mathrm{eV}$. Our exotic scheme corresponds to the choice $m_{1} \approx 10^{-31} \mathrm{eV}$ and $m_{2}>10^{-4} \mathrm{eV}$. In this case, for length scales less than $10^{26} \mathrm{~cm}$ gravity is generated by the exchange of both the massless graviton and the first KK mode. This implies, (taking into account the different coupling suppressions of the massless graviton and the first KK state) that the gravitational coupling as we measure it is related with the parameters of our model as:

$$
\frac{1}{M_{\mathrm{Pl}}^{2}}=\frac{1}{M^{2}}\left(1+\frac{1}{w^{2}}\right) \approx \frac{1}{(w M)^{2}} \Rightarrow M_{\mathrm{Pl}} \approx w M
$$

We see that the mass scale on our brane, $w M$, is now the Planck scale so, although the "warp" factor, $w$, may still be small (i.e. the fundamental scale $M>>M_{\text {Planck }}$ ), we do not now solve even the Planck hierarchy problem. However our example does illustrate how gravity may be quite different from the form that is usually assumed.

Using equations (33) and (21) and assuming as before that $k \approx M$, we find that $m_{1}=$ $2 k e^{-2 x} \approx M_{\text {Planck }} e^{-2 x}$. For $m_{1}=10^{-31} \mathrm{eV}$ we have $m_{2} \approx 10^{-2} \mathrm{eV}$. This comfortably satisfies the bound $m>10^{-4} \mathrm{eV}$ coming from Cavendish experiments. Here we should note that since the coupling of the second mode is always smaller than the one of the first mode, the

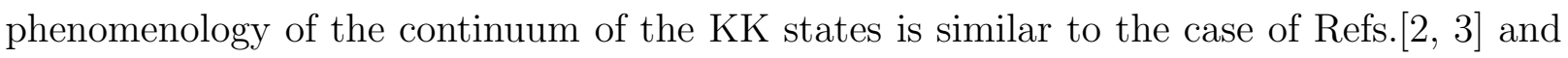
thus does not conflict to experiment.

According to this picture deviations from Newton's law will appear in the submillimeter 


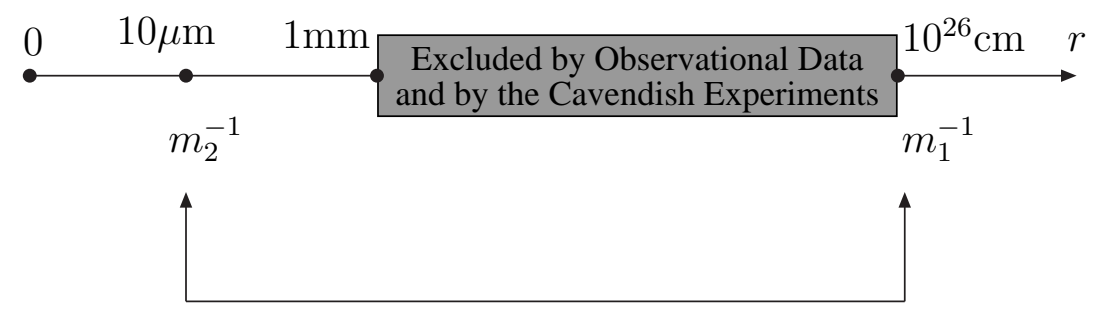

Figure 8: Exclusion regions for the Bi-Gravity case and correlation of the first two KK states

regime as the Yukawa corrections of the second and higher KK states become important. Also the presence of the ultralight first KK state will give deviations from Newton's law as we probe cosmological scales (of the order of the observable universe). The phenomenological signature of this scenario is that gravitational interactions will appear to become weaker by the factor $w$ for distances larger than $10^{26} \mathrm{~cm}$ !

\section{Conclusions}

In this paper we discussed a model of a three 3-brane universe with two positive and one intermediate negative tension brane. Our world is confined to a positive tension brane which makes this construction the minimal realistic model of the RS class. Due to the presence of two positive branes there are now two "bound" states, one associated with the graviton and one with the first KK mode. Compared to the remaining tower of KK states, the latter is has relatively small mass and large coupling. Bounds on the parameter space of this model were placed by comparison with data from collider and Cavendish experiments. However, the phenomenology of the model needs further investigation and it may be that missing energy processes can put stricter bounds on the parameter space.

We also explored the possibility of "Bi-Gravity" in which observable gravity is due to the exchange of both the ordinary graviton and the first ultralight KK state. The novel feature of this description is that gravity is modified at both large and small scales. In particular at large scales the strength of the gravitational force will be reduced by the warp factor. It is clearly of interest to explore the cosmological consequences of such a scheme.

Acknowledgments: S.M., A.P. and J.S. would like to thank Peter B. Renton and Peter Richardson for useful discussions. All authors would like to thank Pavel Kogan for the first three figures and Subir Sarkar for informative discussions. J.S. would like to thank the Department of Theoretical Physics, Oxford University, for a kind hospitality during the course of this project. S.M.'s work is supported by the Hellenic State Scholarship 
Foundation (IKY) No. 8117781027. A.P.'s work is supported by the Hellenic State Scholarship Foundation (IKY) No. 8017711802. J.S.'s work is supported by M.E.C., CICYT (Spain, AEN 96-1672) and Junta de Andalucía (FQM 101). The work of I.I.K. and G.G.R is supported in part by PPARC rolling grant PPA/G/O/1998/00567, the EC TMR grant FMRX-CT-96-0090 and by the INTAS grant RFBR - 950567.

\section{References}

[1] D.Ghilencea and G.G.Ross, hep-ph/9908369.

[2] N. Arkani-Hamed, S. Dimopoulos and G. Dvali, Phys. Lett. B429 (1998) 263.

[3] N. Arkani-Hamed, S. Dimopoulos and G. Dvali, Phys. Rev. D59 (1999) 086004.

[4] I. Antoniadis, N. Arkani-Hamed, S. Dimopoulos and G. Dvali, Phys. Lett. B436 (1998) 257.

[5] L. Randall and R. Sundrum, Phys. Rev. Lett. 83 (1999) 3370.

[6] H. Davoudiasl, J.L. Hewett and T.G. Rizzo, hep-ph/9909255.

[7] S. Chang and M. Yamaguchi, hep-ph/9909523.

[8] I. Oda, hep-th/9908104;

I. Oda, hep-th/9909048.

[9] H. Hatanaka, M. Sakamoto, M. Tachibana and K. Takenaga, hep- th/9909076.

[10] C. Csáki and Y. Shirman, Phys. Rev. D61 (2000) 024008.

[11] T. Li, hep-th/9911234

[12] N. Kaloper, Phys. Rev. D60 (1999) 123506.

[13] L. Randall and R. Sundrum, hep-th/9906064.

[14] J. Lykken, L. Randall, hep-th/9908076.

[15] N. Arkani-Hamed, S.Dimopoulos, G. Dvali and N. Kaloper, hep-th/9907209.

[16] A. Lukas, B.A. Ovrut, K.S. Stelle and D. Waldram, Phys. Rev. D59 (1999) 086001; A. Lukas, B.A. Ovrut and D. Waldram, Phys. Rev. D60 (1999) 086001; hepth/9902071.

[17] N. Kaloper and A. Linde, Phys. Rev. D59 (1999) 101303. 
[18] P. Binétruy, C. Deffayet and D. Langlois, hep-th/9905012.

[19] T. Nihei, Phys. Lett. B465 (1999) 81;

N. Kaloper, Phys. Rev. D60 (1999) 123506.

[20] C. Csáki, M. Graesser, C. Kolda and J. Terning, Phys. Lett. B462 (1999) 34.

[21] J.M. Cline, C. Grojean and G. Servant, Phys. Rev. Lett. 83 (1999) 4245.

[22] H.B. Kim and H.D. Kim, hep-th/9909053.

[23] C. Grojean, J. Cline and G. Servant, hep-th/9910081;

P. Kraus, hep-th/9910149;

A. Kehagias and E. Kiritsis, JHEP 9911 (1999) 022;

T. Shiromizu, K. Maeda and M. Sasaki, gr-qc/9910076;

E. Flanagan, S.H.H. Tye and I. Wasserman, hep-ph/9910498;

D. Ida, gr-qc/9912002.

[24] P. Kanti, I.I. Kogan, K.A. Olive and M. Pospelov, Phys. Lett. B468 (1999) 31;

P. Kanti, I.I. Kogan, K.A. Olive and M. Pospelov, hep- ph/9912266.

[25] C. Csáki, M. Graesser, L. Randall, and J. Terning, hep-ph/9911406.

[26] P. Horava, and E. Witten, Nucl. Phys. B460 (1996) 506; B475 (1996) 94.

[27] W.D. Goldberger and M.B. Wise, Phys. Rev. D60 (1999) 107505;

W.D. Goldberger and M.B. Wise, Phys. Rev. Lett. 83 (1999) 4922;

W.D. Goldberger and M.B. Wise, hep-ph/9911457.

[28] O. De Wolfe, D.Z. Freedman, S.S. Gubser and A. Karch, hep-th/9909134.

[29] M.A. Luty and R. Sundrum, hep-th/9910202.

[30] T. Han, J.D. Lykken and R.J. Zhang, Phys. Rev. D59 (1999) 105006. 